\title{
The prevalence of hyperfibrinolysis detected by rotational thromboelastometry in neurosurgical patients with traumatic brain injury (TBI) requiring craniotomy and hematoma evacuation
}

\author{
M. Rimaitis ${ }^{1}$, D. Bilskiene ${ }^{1}$, T. Tamosuitis ${ }^{2}$, R. Vilcinis ${ }^{2}$, A. Macas ${ }^{1}$ \\ ${ }_{1}^{1}$ ithuanian University of Health Sciences, Clinic of Anesthesiology \\ 2Lithuanian University of Health Sciences, Clinic of Neurosurgery
}

Background and Goal of Study: Hyperfibrinolysis is proposed as one of the mechanisms leading to coagulation substrate consumption and excessive blood loss in trauma patients. Consequently, the use of antifibrinolytic agents has become widespread. However, the efficacy and possible benefits of their use in TBI patients remains debated. Viscoelastic assays are increasingly used to detect, quantify fibrinolysis, and predict the potential effect of antifibrinolytic agent administration. We aimed to clarify the prevalence of hyperfibrinolysis using thromboelastometry (ROTEM) in isolated TBI patients, and to evaluate the potential effect of antifibrinolytic agent administration.

Materials and Methods: We performed a prospective, observational pilot study in a cohort of adult TBI patients requiring urgent craniotomy due to intracranial hypertension. Patients with multiple trauma and preoperative exposure to antifibrinolytics, were not included. Isolated severe TBI was defined as Abbreviated Injury Scale for head $\left(\right.$ AIS $\left._{\text {head }}\right)>3$, and AIS $_{\text {extracranial }}<3$. Blood was collected preoperatively and analyzed with ROTEM assays (EXTEM, INTEM, FIBTEM and APTEM). Coagulopathy was defined as any abnormality on ROTEM. Hyperfibrinolysis was defined as ML $>15 \%$ on EXTEM, INTEM or FIBTEM, and/or better APTEM parameters in presence of pathologic EXTEM readings. EXTEM and APTEM results were compared. Data are expressed as median (Q1Q3).

Results and Discussion: In a cohort of 33 patients (Glasgow coma score: 7(5-11.5); midline shift $>0.5 \mathrm{~cm}$ on CT in $75 \%$ cases; ASA status $>=3$ in $97 \%$ cases), abnormal preoperative coagulation pattern according to ROTEM was found in $10(30.3 \%)$ patients. Hyperfibrinolysis was found in $2(6.1 \%)$ patients (figure 1$)$. Of note, overall coagulation pattern on APTEM was better in the majority of patients 18 (54.5\%), but the difference was only slight: CT 58(5372) vs $59(54-78)$ s; CFT $88(64-120.5)$ vs $85(72.5-127) s$, MCF $60(53-65.5)$ vs $62(53.5-65.5) \mathrm{mm}$ and ML 5(3-6.5) vs $4(2.5-6) \%$ in EXTEM and APTEM, respectively. Statistically significant difference between EXTEM and APTEM was found only with regard to maximum lysis (ML), $p<0.05$. However $\mathrm{ML}_{\text {EXTEM }}$ was $<15 \%$ in all cases (table 1 ).
Table 1. Preoperative coagulation parameters of study cohort

Standard Laboratory tests

\begin{tabular}{|c|c|c|c|c|c|c|}
\hline $\begin{array}{c}\mathbf{H b}, \\
\mathbf{g} / \mathbf{d L}\end{array}$ & $\begin{array}{c}\mathbf{H c t ,} \\
\mathbf{\%}\end{array}$ & $\begin{array}{c}\mathbf{P l t}, \\
\mathbf{1 0} / \mathbf{L}\end{array}$ & $\begin{array}{c}\text { PTI, } \\
\mathbf{\%}\end{array}$ & $\begin{array}{c}\text { INR } \\
\text { APTT, } \\
\mathbf{S}\end{array}$ & $\begin{array}{c}\text { Fibr., } \\
\mathbf{g / L}\end{array}$ \\
\hline 133 & 38.8 & $\begin{array}{c}195 \\
(121.5-145)\end{array}$ & $\begin{array}{c}93 \\
(35.9-41.5)\end{array}$ & $\begin{array}{c}1,03 \\
(147.5-256)\end{array}$ & $\begin{array}{c}(79.5-110.5) \\
(0.96-1.1)\end{array}$ & $\begin{array}{c}(28.9-33.5) \\
(2.18-4.1)\end{array}$ \\
\hline
\end{tabular}

Thromboelastometry

\begin{tabular}{|c|c|c|c|c|c|c|}
\hline & $C T, s$ & CFT, $s$ & $\begin{array}{l}\text { alpha } \\
\text { angle }\end{array}$ & A10, $\mathbf{m m}$ & MCF, $\mathbf{m m}$ & $M L, \%$ \\
\hline INTEM & $\begin{array}{c}155 \\
(139.5-174.5)\end{array}$ & $\begin{array}{c}86 \\
(65-106)\end{array}$ & $\begin{array}{c}74 \\
(70-77)\end{array}$ & $\begin{array}{c}51 \\
(46-59)\end{array}$ & $\begin{array}{c}60 \\
(55-65)\end{array}$ & $\begin{array}{c}4 \\
(2-7)\end{array}$ \\
\hline EXTEM & $\begin{array}{c}58 \\
(53-72) \\
\end{array}$ & $\begin{array}{c}88 \\
(64-120.5) \\
\end{array}$ & $\begin{array}{c}74 \\
(67-78) \\
\end{array}$ & $\begin{array}{c}52 \\
(45-60)\end{array}$ & $\begin{array}{c}60 \\
(53-65.5) \\
\end{array}$ & $\begin{array}{c}5 * \\
(3-6.5) \\
\end{array}$ \\
\hline APTEM & $\begin{array}{c}59 \\
(54-78) \\
\end{array}$ & $\begin{array}{c}85 \\
(72.5-127) \\
\end{array}$ & $\begin{array}{c}74 \\
(68.5-77) \\
\end{array}$ & $\begin{array}{c}54 \\
(45-60) \\
\end{array}$ & $\begin{array}{c}62 \\
(53.5-65.5) \\
\end{array}$ & $\begin{array}{c}4 * \\
(2.5-6) \\
\end{array}$ \\
\hline FIBTEM & $\begin{array}{c}60 \\
(47.5-73.5) \\
\end{array}$ & NA & NA & $\begin{array}{c}19 \\
(13-21) \\
\end{array}$ & $\begin{array}{c}21 \\
(14.5-24.5) \\
\end{array}$ & $\begin{array}{c}0 \\
(0-3)\end{array}$ \\
\hline \multicolumn{7}{|c|}{$\begin{array}{l}\text { Sata are expressed as median (Q1-Q3). } \\
\text { Significant difference according to Related-samples Wilcoxon test, } \mathrm{p}<0.05 \text {. } \\
\text { tb - hemoglobin concentration, HCt - hematocrit, Plt - platelet count, PTI - prothrombin time index, INR - } \\
\text { nternational normalized ratio, APT - activated partial thromboplastin time, Firr. -fibrinogen concentration. } \\
\text { TT - clotting time, CFT - clot formation time, A10 - clot amplitude after } 10 \text { minutes, MCF - maximum clot } \\
\text { irmness, ML - maximum lysis. }\end{array}$} \\
\hline
\end{tabular}

Figure 1. Significant improvement of clot quality in APTEM assay as compared with EXTEM in a patient with severe TBI
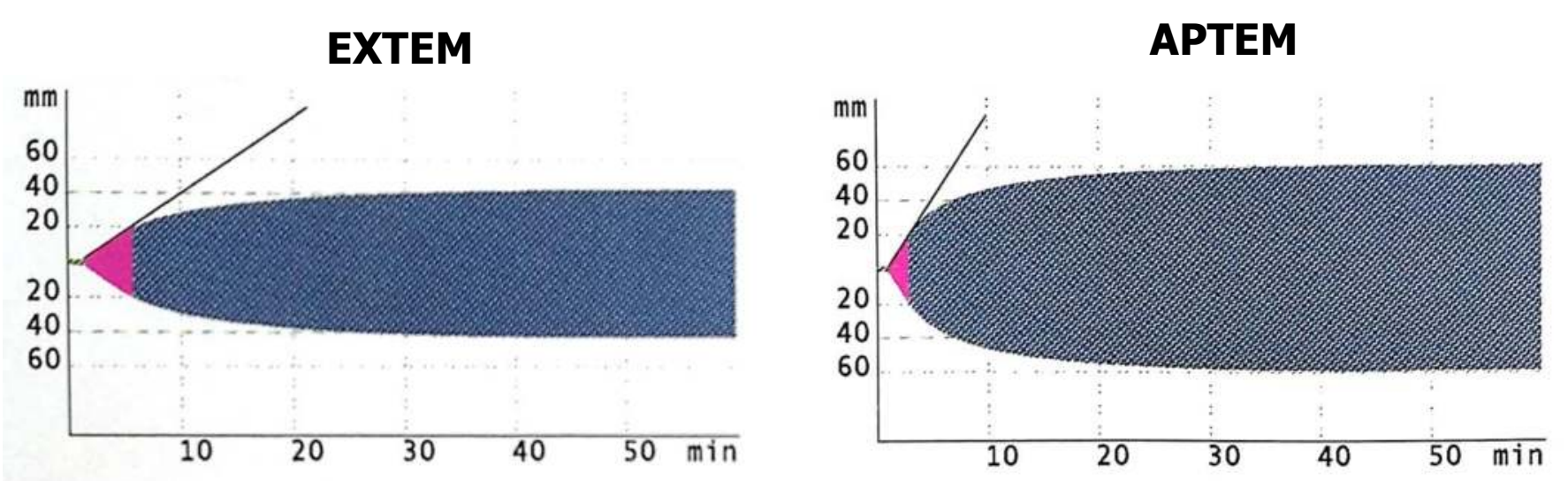

\begin{tabular}{|c|c|c|c|c|c|c|c|c|c|c|c|}
\hline CT & : & 94 & $S$ & 38 & & 79] & $\Delta$ & CT & : & 66 & S \\
\hline CFT & : & 256 & S & 34 & - & 159] & $\Delta$ & CFT & : & 110 & $\mathrm{~s}$ \\
\hline MCF & : & 43 & $\mathrm{~mm}$ & 50 & - & 72] & $\nabla$ & MCF & : & 59 & $\mathrm{~mm}$ \\
\hline ML & $:{ }^{*}$ & $\begin{array}{lll}* & 0\end{array}$ & $\%$ & 0 & - & 15] & & ML & * & 1 & $\%$ \\
\hline$\alpha$ & : & 49 & 。 & 63 & - & 83] & $\nabla$ & $\alpha$ & : & 69 & 。 \\
\hline A5 & : & 22 & $\mathrm{~mm}$ & 34 & - & 55] & $\nabla$ & A5 & : & 38 & $\mathrm{~mm}$ \\
\hline $\mathrm{A} 10$ & : & 31 & $\mathrm{~mm}$ & 43 & - & 65] & $\nabla$ & A10 & : & 48 & $\mathrm{~mm}$ \\
\hline
\end{tabular}

Conclusion: Hyperfibrinolysis might be not as prevalent as expected in isolated TBI patients. The role of antifibrinolytic agents in brain trauma is yet to be defined.

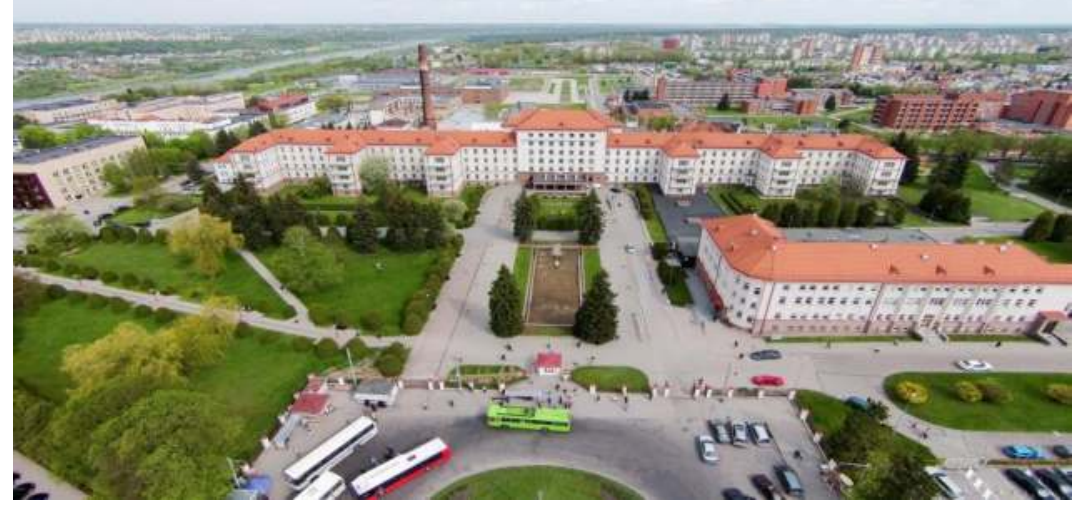

